\title{
Attribution Inference for Digital Advertising using Inhomogeneous Poisson Models
}

\author{
Zachary Nichols* \\ Spotify, Inc. \\ New York, NY \\ zack@spotify.com
}

\author{
Adam Stein \\ Spotify, Inc. \\ New York, NY \\ astein@spotify.com
}

\begin{abstract}
Measuring the causal effect of advertising on driving desired behavior is an important problem to the digital publishing industry (the "attribution" problem) [3, 9]. It is common to use observational methods for attribution, due to the high cost and difficulty of employing randomized controlled trials (RCTs). However, recent results have shown that even current sophisticated observational methods may be inaccurate, yielding incorrect estimates of the true effect of advertising [4]. Here, we present a new observational attribution method based on a successful model of neural spiking $[11,13]$ that learns the temporal interactions between event-based time series. We train this model on data from several RCT marketing experiments, and show that it can accurately recover the true causal attribution.
\end{abstract}

\section{ACM Reference Format:}

Zachary Nichols and Adam Stein. 2018. Attribution Inference for Digital Advertising using Inhomogeneous Poisson Models. In WWW 2018: The 2018 Web Conference, April 23-27, 2018, Lyon, France. ACM, New York, NY, USA, 8 pages. https://doi.org/10.1145/3178876.3186171

\section{INTRODUCTION}

Digital advertising is a critically important source of revenue for many Internet-based companies. This revenue has been growing year over year, with spending on digital advertising in the US forecasted to reach $\$ 83$ billion in 2017 [7]. As this spending has increased, so has the demand to accurately measure the performance of advertising [1] - the "attribution" problem.

While in principle the attribution problem can be solved with randomized controlled trials (RCTs), practical issues mean it is often more common to use observational methods [4]. One major such issue is that measuring attribution with RCTs requires very large sample sizes to yield reliable estimates [8]. This drawback becomes compounded when considering "multi-touch attribution": the problem of estimating causal effects under the common scenario where users are shown ads across multiple platforms and channels (such as search, display, or social ads). Coordinating multiple RCTs across several channels and formats is difficult or unrealistic for many advertisers, and falling back to observational methods is natural.

"current affiliation: Stitch Fix, Inc., <zack.nichols@stitchfix.com>

This paper is published under the Creative Commons Attribution 4.0 International (CC BY 4.0) license. Authors reserve their rights to disseminate the work on their personal and corporate Web sites with the appropriate attribution.

WWW 2018, April 23-27, 2018, Lyon, France

() 2018 IW3C2 (International World Wide Web Conference Committee), published under Creative Commons CC BY 4.0 License.

ACM ISBN 978-1-4503-5639-8/18/04

https://doi.org/10.1145/3178876.3186171
A big limitation of observational methods though is that they can be inaccurate. Several studies examining common observational attribution approaches have demonstrated this inaccuracy. Lewis et al. [10] used large-scale RCTs conducted at Yahoo to show that position-based attribution methods - such as "last touch", which awards credit to the ad that a user saw most recently before a conversion - fail to capture the true ad effects. Sapp et al. used simulations to demonstrate that several methods for inferring attribution fail to recover the true effect of advertisements in typical scenarios, including both position-based attribution methods [15] and two data-driven methods: user matching and propensity scoring [14]. Gordon et al. [4], in a recent thorough study, evaluated multiple data-driven attribution methods against 15 RCT experiments conducted at Facebook, and found that they often fail to recover the true effects.

While these studies demonstrated that some of the most common approaches for data-driven observational attribution fail to recover the true effects of digital advertising, it is still possible in principle that other methods could overcome this issue. One type of approach that has seen significant attention, but not yet been evaluated by RCTs (to the knowledge of the authors), is the use of probabilistic models that are built to leverage temporal information. Examples include the use of survival theory [21], Hawkes process models [20], and hybrids of the two [5].

Here, we present a new observational attribution method that is designed to primarily take advantage of temporal information. It is based on a successful family of models used previously to characterize coupled neuronal systems [11,13], and treats conversion events as arrivals in an inhomogeneous Poisson point process, the rate of which is modulated by the arrival of ad impressions. This approach has similarities with the use of above-mentioned temporal models. It differs in substantial ways, however. Rather than assuming a fixed functional shape for the temporal interactions, (e.g. exponential), this model learns the shape of a temporal filter from the data directly. It also learns the dilution of expected conversion yield over repeated impressions.

To validate this model, we ran several multivariate randomized controlled trials on the Spotify music streaming platform. These trials were run on campaigns for Spotify subscription products that took advantage of multiple channels: native in-app ads, ads placed between pieces of streaming content, and emails. A comparison with RCT holdout groups shows that the observational approach presented here accurately recovers the true causal attribution from advertising in a multi-channel setting.

We also compare this method to a simpler inhomogeneous Poisson model whose temporal filters consist of exponential functions. While the exponential model (comparable to a Hawkes Process or 
survival model approach $[5,20,21])$ is not as accurate as the full model with learned temporal filters, in several cases it performs reasonably well at recovering causal attribution. The performance of these models, in contrast to the previously-reported results with other data-driven observational methods, suggest that impression and conversion timing is a highly informative factor that should not be overlooked for observational attribution methods.

\section{METHODS}

\subsection{Measuring Temporal Correlations}

We begin the analyses in this report by first characterizing the temporal relationship between ad impressions and conversion events using covariograms $[2,12]$. This way we can visualize and quantify the degree and timescale of correlated activity between events, and use this information to guide model choices. Since this method is not common in the advertising effectiveness literature, we provide some details here.

Specifically, for a given user $u$ and advertisement $a$, we let $\psi_{u, a}(i)$ be the time of the user's $i$ th impression of the ad. Similarly, we let $\omega_{u}(j)$ be the time of the users $j$ th conversion event (when the user engaged in the desired behavior). Note that while this notation and the following model formulation is general enough to allow for an arbitrary number of conversions, for many applications (including the data analyzed in this report), there will be at most 1 conversion event.

We let $r_{u, a}(t)$ and $y_{u}(t)$ contain the binned time series for the ad impressions and conversion events (respectively): $r_{u, a}(t)=$ $\sum_{i} \mathbb{1}_{0}\left\lfloor\frac{t-\psi_{u, a}(i)}{b}\right\rfloor ; y_{u}(t)=\sum_{j} \mathbb{1}_{0}\left\lfloor\frac{t-\omega_{u}(j)}{b}\right\rfloor$, where $\mathbb{1}_{0}$ is an indicator function that maps 0 to 1 and all other values to 0 , and $b$ is the bin size.

The cross-correlogram between ad impressions and conversions for a user is given by (where $\star$ denotes the cross-correlation operator, and $\tau$ represents a time offset between events):

$$
\left(r_{u, a} \star y_{u}\right)(\tau)=\int_{-\infty}^{\infty} r_{u, a}(t) y_{u}(t+\tau) d t
$$

Letting \langle\rangle represent averaging across users in the population, we get the estimated "raw" correlogram for the ad: $R_{a}(\tau)=\left\langle r_{u, a} \star y_{u}\right\rangle$.

This raw correlogram is driven both by the effects of ads themselves, and from any other effects that might happen at the same time (such as from ads in non-digital media, or spreading of information via word-of-mouth). We subtract some of these latter non-local effects by using a "shuffle predictor", borrowed from neuroscience [2,12]. It is given by $K_{a}(\tau)=\left\langle r_{u, a}\right\rangle \star\left\langle y_{u}\right\rangle$. Note that the shuffle predictor cannot contain any local effects, and is equivalent to the expected correlogram you would get if conversion outcomes were randomly shuffled between users.

Subtracting the shuffle predictor from the raw correlogram gives the corrected covariogram $V_{a}(\tau)$, which we use to estimate the temporal correlation between advertisements and conversion events:

$$
V_{a}(\tau)=R_{a}(\tau)-K_{a}(\tau)=\left\langle r_{u, a} \star y_{u}\right\rangle-\left\langle r_{u, a}\right\rangle \star\left\langle y_{u}\right\rangle .
$$

\subsection{Conversion Model Formulation}

The correlograms provide a characterization of the temporal interaction between events, but do not themselves allow for predictions
Table 1: Notation

\section{Symbol Description}

\begin{tabular}{ll}
$u, a$ & user, advertisement \\
$\psi_{u, a}(i)$ & the time of user $u$ 's $i$ th impression of ad $a$ \\
$\omega_{u}(j)$ & the time of user $u$ 's $j$ th conversion event \\
$\left|\omega_{u}\right|$ & the number of conversions for user $u$ (typically $\leq 1)$ \\
$r_{u, a}(t)$ & user $u$ 's binned impression rate for ad $a$ at time $t$ \\
$y_{u}(t)$ & user $u$ 's binned conversion rate at time $t$ \\
$\lambda_{u}(t)$ & user $u$ 's model conversion rate at time $t$ \\
$\beta$ & user feature regression weights \\
$x_{u}$ & user $u$ 's relevant features \\
$d_{a}(i)$ & ad $a$ 's discounted impact for its $i$ th impression \\
$h_{a}(\tau)$ & ad $a$ 's temporal coupling filter \\
\hline
\end{tabular}

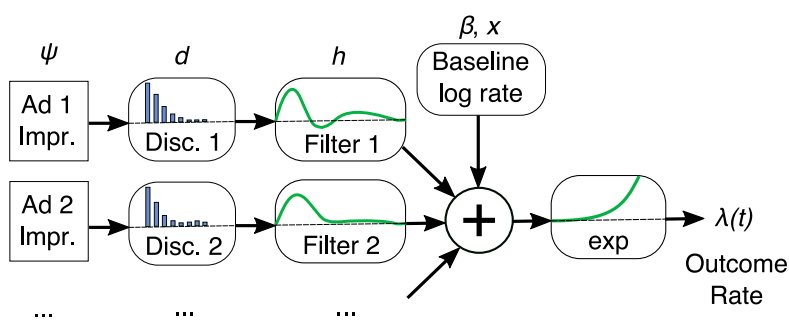

Figure 1: A schematic of the inhomogeneous Poisson conversion model. Ad impressions $(\psi)$ are assigned a value by a discount function $(d)$ that accounts for repeated impressions. The discounted impression is then fed through a temporal linear filter $(h)$ that, together with the other filter outputs and baseline rate $(\beta \cdot x)$, is fed through a static exponential nonlinearity to produce the instantaneous conversion rate $\lambda(t)$.

or for attribution. For that, we formulate a probabilistic model of conversion based on the received temporal sequence of ad impressions. Our approach (see diagram in fig. 1) is inspired by models of coupled neuronal firing $[11,13]$, with a new addition of discount factor terms that model the fatigue from repeated impressions.

Specifically, we model the arrival of a user $u$ 's conversion events $\left(\omega_{u}\right)$ with an inhomogeneous Poisson process with time-varying rate $\lambda_{u}(t)$

We let $\lambda_{u}(t)$ vary in time according to exposure to ads $\left(\psi_{u, a}\right)$ via the formula (where $*$ denotes the convolution operator):

$$
\begin{aligned}
\lambda_{u}(t) & =\exp \left[\beta \cdot x_{u}+\sum_{a} \sum_{i} d_{a}(i) h_{a}\left(t-\psi_{u, a}(i)\right)\right] \\
& =\exp \left[\beta \cdot x_{u}+\sum_{a}\left(s_{u, a} * h_{a}\right)(t)\right] .
\end{aligned}
$$

This formula consists of a linear function (the terms inside of the exponentiation) which is fed through a static exponential nonlinearity. The linear portion can be further broken down into a constant portion and a time-varying portion. The constant portion consists 
of the dot product term $\beta \cdot x_{u}$, where $x_{u}$ is a vector containing user-level features (data used in this report contains country of residence as the lone user-level feature), and $\beta$ is a learned set of weights which are applied to them before summing.

The time-varying portion responds to ads, and is given by the term to the right of the ' + ' sign. It is written two equivalent ways here. The first version explains the time-varying component as a sum of terms of an impression's discount factor $d_{a}(i)$ (which accounts for repeated impressions) multiplied by the value of the ad's temporal filter $h_{a}$ at relative time since the impression, $t-\psi_{u, a}(i)$ The second version is written in an equivalent but more compact notation that demonstrates the convolution relationship between the ad filters $h_{a}$ and a discount-weighted series of impressions $s_{u, a}$, given by $s_{u, a}(t)=\sum_{i} d_{a}(i) \mathbb{1}_{0}\left\lfloor\frac{t-\psi_{u, a}(i)}{b}\right\rfloor$. See Results for examples of linear output for a model fit on experimental data.

We observe events over a finite time window, with $t=0$ labelling to the beginning of the window, and $t=t_{e}$ labelling the end of the window. For datasets used in this report, a conversion event means that a user no longer receives advertisements, and the window is over. For users who do not convert, the window extends to the end of the campaign.

Our goal is to learn model parameters $\Theta=\left\{\beta_{1}, h_{1}, d_{1}, \ldots\right\}$. We do this by using the standard continuous-time formula for the probability of a Poisson process realization (letting $\Psi_{u}$ be the set of ad impressions for the user $\left.\left\{\psi_{u, 1}, \psi_{u, 2}, \ldots\right\}\right)$ :

$$
\log P\left(\omega_{u}, \Psi_{u} \mid \Theta\right)=\sum_{j} \log \lambda_{u}\left(\omega_{u}(j)\right)+\int_{t=0}^{t=t_{e}} \lambda_{u}(t) d t .
$$

We find a maximum a-posteriori (MAP) estimate of the parameters $\Theta$ by using equation 3 with Bayes' rule, $P(\Theta \mid \omega, \Psi) \propto$ $P(\omega, \Psi \mid \Theta) P(\Theta)$, yielding:

$$
\hat{\Theta}=\arg \max _{\Theta} \sum_{u} \log P\left(\omega_{u}, \Psi_{u} \mid \Theta\right)+\log P(\Theta) .
$$

We use weak Gaussian $\left(l_{2}\right)$ priors for the $\beta$ and $h$ parameters, and use an improper uniform prior for the $d$ parameters.

To reduce the size of the parameter space, we decompose the coupling filters into a linear combination of basis vectors according to $h_{a}=\mathbf{B} k_{a}^{\top}$, where $\mathbf{B}$ is a basis matrix, and $k_{a}$ (the "kernel") is the vector of weights used to construct the filter $h_{a}$. For the full conversion model, we use a raised cosine basis with logarithmic spacing (following Pillow et al. [13]). We also use the covariogram calculations from equation 1 to determine the necessary size of the coupling filters and the appropriate temporal bin size $b$.

If we consider only the $\beta$ and $h$ parameters of this model, holding $d$ fixed, this reduces to a type of Generalized Linear Model (GLM), with a convex likelihood function [11]. Jointly considering the $d$ parameters yields a more complicated optimization, by effectively adding a matrix factorization, so that the model is technically no longer convex. In practice however, adding this type of matrix factorization to a "GLM" is common and yields good results [18].

\subsection{Attribution Measurement}

We use data from both the probabilistic conversion models and RCTs to compute measures of causal attribution.
We define the conversion rate for a group of users $G$ as $\mu_{G}=$ $\mathbb{E}_{u \in G}\left[\left|\omega_{u}\right|\right]$, and the "counterfactual" [3] conversion rate under another hypothetical regime of ad exposure as $\mu_{g}^{C}=\mathbb{E}_{u \in G}\left[\left|\omega_{u}^{C}\right|\right]$. We measure attribution as the difference between these two conversion rates: $\Delta_{G}=\mu_{G}-\mu_{G}^{C}$.

For an RCT, we can let $G$ be a treatment group (exposed to ads), and estimate $\hat{\mu}_{G}=\frac{1}{|G|} \sum_{u \in G}\left|\omega_{u}\right|$. The counterfactual conversion rate can be directly estimated from the control group $G_{\text {ctrl }}$ as $\hat{\mu}_{G}^{C}=\frac{1}{\left|G_{\text {ctrl }}\right|} \sum_{u \in G_{\text {ctrl }}}\left|\omega_{u}\right|$. This yields the estimate $\hat{\Delta}_{G}=\hat{\mu}_{G}-\hat{\mu}_{G}^{C}$. Since for our experiments conversion can happen at most once per user, we can use the standard normal approximation to the binomial to compute the standard error of this estimator: $\operatorname{SE}\left[\hat{\Delta}_{G}\right]=$ $\sqrt{\hat{\mu}_{G}\left(1-\hat{\mu}_{G}\right) /|G|}+\sqrt{\hat{\mu}_{G}^{C}\left(1-\hat{\mu}_{G}^{C}\right) /\left|G_{\text {ctrl }}\right|}$.

For a model-based attribution measurement, we estimate $\hat{\mu}_{G}$ in the same way as the RCT, but we do not make use of the counterfactual conversion rate. Instead, we simulate the expected number of conversions under the counterfactual ad exposure regime:

$$
\hat{\mu}_{G}^{C}=\frac{1}{|G|} \sum_{u \in G} \int_{t=0}^{t=t_{e}} \exp \left[\lambda_{u}^{C}(t)\right] d t
$$

where $\lambda_{u}^{C}(t)$ is the hypothetical Poisson conversion rate under the counterfactual delivery regime, given by feeding the hypothetical ad delivery into eq. 2 . In the case where the counterfactual regime includes no advertisements (or includes ads of no expected effect such as Public Service Announcements), this reduces to:

$$
\hat{\mu}_{G}^{C}=\frac{1}{|G|} \sum_{u \in G} \int_{t=0}^{t=t_{e}} \exp \left[\beta \cdot x_{u}\right] d t
$$

\subsection{Experiments}

We conducted randomized controlled trials on the Spotify music streaming platform. Spotify has both a subscription-based tier and an ad-supported free tier, and a portion of the advertising on the free tier is for its own subscription products. This means it acts as both an advertiser and publisher, which affords large degree of control and visibility over both delivering ads and measuring the eventual conversion events.

We used this platform to conduct three RCT experiments, assessing the causal impact of advertising across three channels (incontent ads, in-app native ads, and e-mails) in driving conversion events.

Experiment 1 involved advertising an introductory promotional product to English-speaking users in North America, over 40 days in the summer of 2017. Treatment arms included a control group (who received only generic advertising rather than promotions), a group which received in-app native ad promotions, a group which in-app ad promotions and email only, and finally a group which received in-app ads, email, and in-content promotional ads. Users were randomly assigned to the groups at the beginning of the campaign, with slightly over 425,000 users per group.

Experiment 2 had an identical construction to Experiment 1, but involved users in rapidly-growing Spanish- and Portuguesespeaking markets. Users were again randomly assigned to groups, with about 175,000 users per group.

Experiment 3 involved advertising a new type of subscription product to users in the EU, Canada, Brazil, and Mexico, over 14 
A

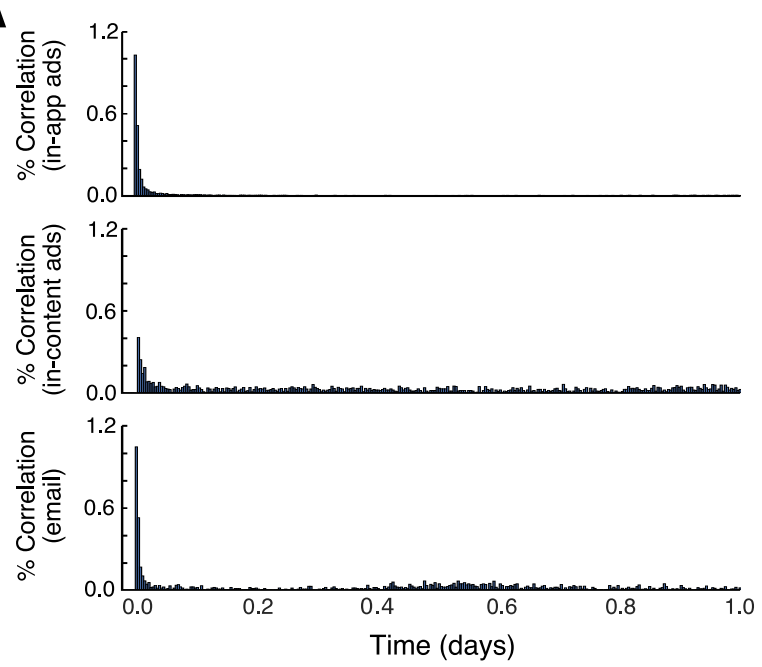

B

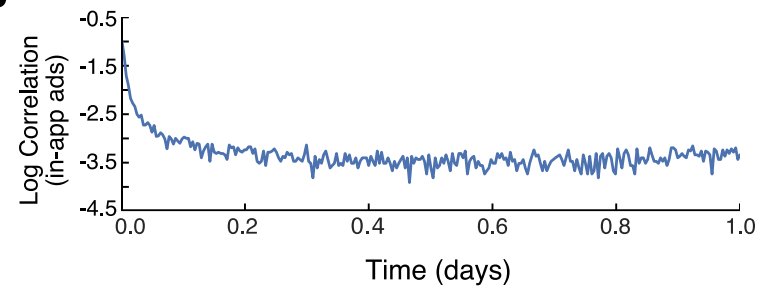

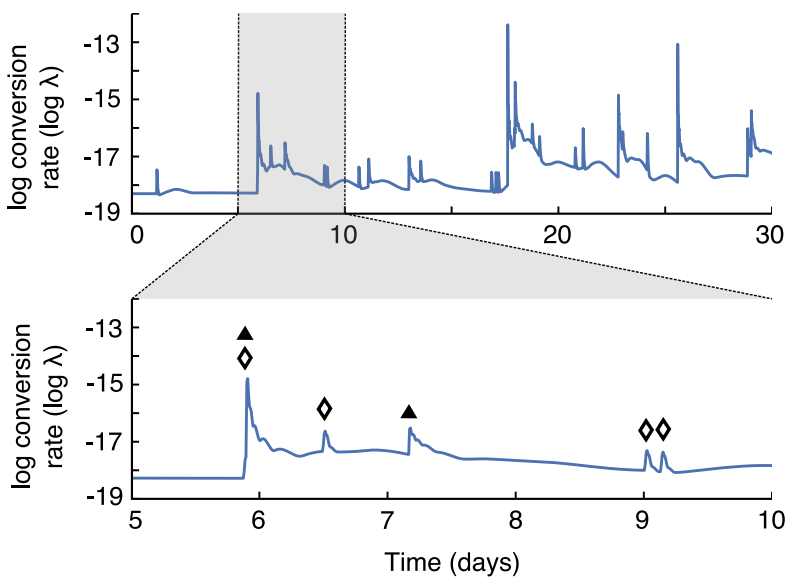

Figure 3: Sample traces of the linear model output for a randomly-chosen example user in Experiment 1. The linear output (the $\log$ of $\lambda(t)$ as given by eq. 2) over 30 days is shown in the top trace. A 5-day section is expanded in the bottom trace, with individual impressions labelled. Diamonds correspond to in-content ads, and triangles correspond to in-app ads. In both traces we can see that ads have a large effect on the linear output, with a non-trivial temporal structure. $C f$. correlograms in fig. 2 , vs. traces in previous reports ([21] fig. 2 , [20] fig. 2).

plotted over a sample range of 24 hours are shown in fig. 2 for the advertising channels studied here: in-app ads, email, and incontent ads. The strongest correlation between impression time and conversion is immediate, but weaker correlations exist at longer timescales, and may have a complicated structure. For example, there is a non-trivial amount of correlation between email impressions and conversion events at a lag of 12 hours (panel A, bottom).

Even ads with an apparently simpler correlation structure, such as in-app ads (fig. 2 A, top), may not be well-described by a single exponential decay. Fig. $2 \mathrm{~B}$ shows the correlogram for in-app ads on a log scale. We can see a very non-linear drop in the degree of correlation on short timescales, as opposed to the linear drop we would expect from an exponential decay.

\subsection{Fitting Nonhomogeneous Poisson Models to Experiment Data}

The timescales of the temporal interactions described by the corrected covariograms are used to determine the necessary width of the temporal kernels used in the conversion model. A schematic of the conversion model for a single user is shown in fig. 1, and it is fully described in the Methods. Briefly, the model contains a feed-forward structure with five steps. First, impressions for each advertisement are given a weight according to how many times they have already been seen by the user. Next, this weight is used to scale a temporal filter for the advertisement that describes its effect on conversion probability. The results of these filters across the advertisements are added together, along with a baseline log conversion rate for the user. This linear output is then converted 
to a probability by exponentiating the results. Finally, this output is used as a conversion rate in an inhomogeneous Poisson process.

Data from the three experiments was used to fit conversion models. For Experiment 1 and Experiment 2, the control group received generic advertising and the treatment groups received advertising for a special promotional product. For Experiment 3, the control group received no advertising of expected impact. To fit the models for Experiments 1 and 2 therefore, data from a random $5 \%$ of the control group was included in training (to learn the model components for the generic ads), with evaluation performed on the remaining 95\%. For Experiment 3, no data from the control group was included in training.

Once fit, the models can be used to simulate conversion events, or to calculate the expected number of conversions (via eq. 5). Example traces of the linear output which is used to make these calculations is shown in fig. 3. Comparing the output traces to fig. 2 shows that the inhomogeneous Poisson model with learned filters is able to capture some of the complicated correlation structure that exists in the data. This is especially clear comparing to fig. $2 \mathrm{~B}-$ the exponential filters used in previous temporal models would appear as straight lines on both plots.

\subsection{Evaluating Model Performance for Attribution}

We next evaluate the performance of the model at its intended task: inferring attribution. We do this by comparing the model-derived attribution to the values given by the RCT comparisons. Results for each of the treatment groups is shown in fig. 4. In each panel, we present the attribution $\Delta$ (see Methods), estimated both with the RCT (blue) and by the inhomogeneous Poisson model (green). Confidence intervals for the attribution $\Delta$ are calculated using Standard Error under a normal approximation to binomial outcomes. We do not calculate a measure of uncertainty for the model-derived attribution here, instead treating it as a point estimate which we can compare to the RCT-derived CI.

The inhomogeneous Poisson model was able to closely match the "true" attribution for each treatment group in each of the three experiments. We gauge accuracy by the how closely the model agrees with the result from the RCT. As is shown, the model-based attribution is inside the $95 \%$ confidence interval for the RCT for each treatment group. This is the case both when the measured attribution is high (Experiments 1 and 2) and when it is very low (Experiment 3; note the y-axis scale in fig. $4 \mathrm{C}$ ). While in principle we could have found significant differences between the model and RCT results with a larger sample size, such as was employed in some previous studies of attribution [4] (since this would result in narrower confidence intervals), we note that the model is consistently within a fraction of a percentage point of the RCT results.

In order to gauge how important the flexible temporal coupling filters are for the model's accuracy (as compared to the exponential functions assumed by Hawkes Process models), we also fit a limited version of the inhomogeneous Poisson that was restricted to filters with a linear shape (which become exponential when fed through the fixed nonlinearity), using data from Experiment 1 . The results are shown in fig. 5 . The exponential version of the model is not
A

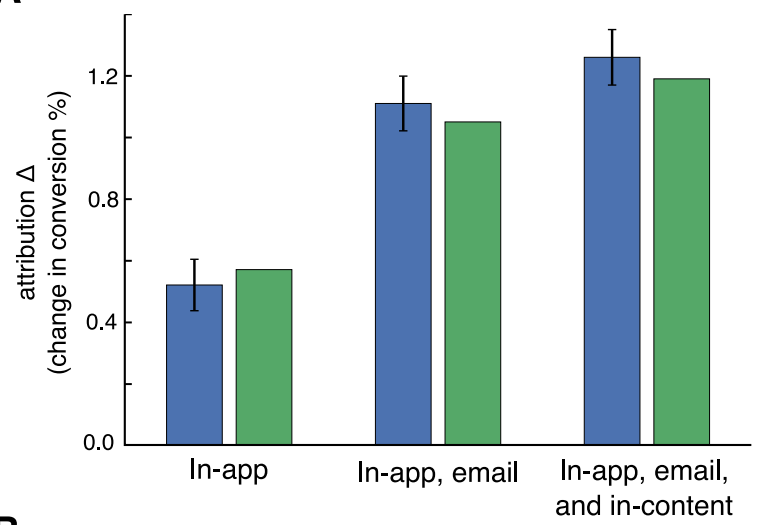

B

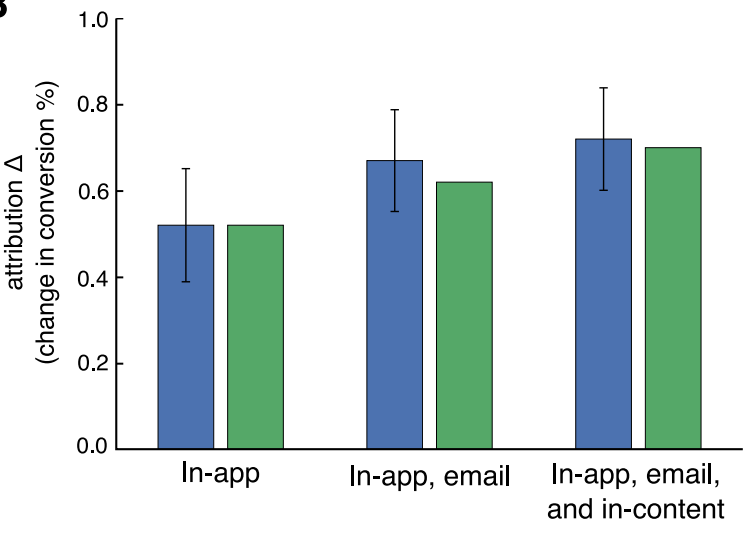

C

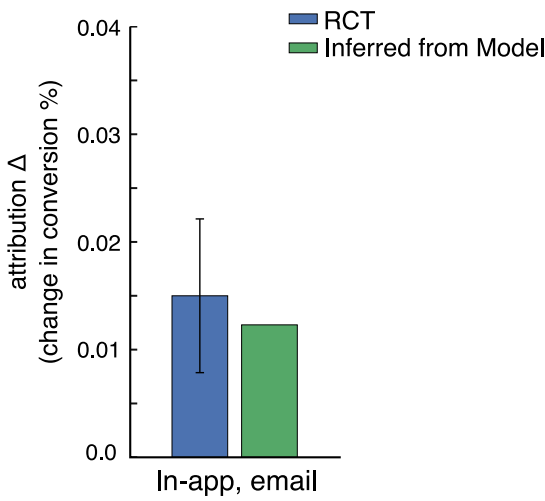

Figure 4: Performance of the Inhomogeneous Poisson conversion model in inferring attribution. For each treatment group, the RCT-inferred attribution (blue) is determined by comparing the conversion rate from the treatment group vs the control hold-out group (which received no advertisements). Error bars represent $95 \%$ confidence intervals. The model-inferred attribution ( $g r e e n$ ) is determined by training the conversion model on the each treatment group alone, and using eq. 5 to estimate the control group conversion rate. The model is able to accurately recover the true advertising effects even when multiple channels are used for advertisement. Results are shown for all treatment groups in Experiment 1 (Panel $A$ ), Experiment 2 (Panel $B)$, and Experiment 3 (Panel C). 


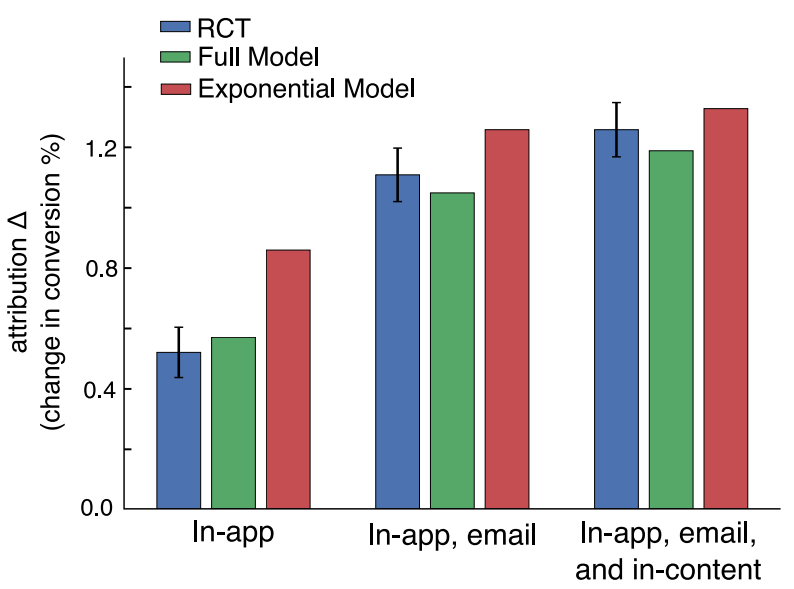

Figure 5: Comparison of the "full" Inhomogeneous Poisson model (using temporal filters with a learned shape) vs. a model where temporal kernels are restricted to exponential functions, as in a Hawkes process. While the exponential model ( $r e d)$ yields attribution estimates close to the full model (green) and the RCT (blue) in two out of three cases, it yields an overestimate for the treatment group that only received in-app advertisements (unlike the full model).

as accurate as the full version, and the attribution estimates fall outside of the $95 \%$ confidence intervals from the RCT for two of the examined treatment groups.

\section{DISCUSSION}

\subsection{Temporal information is important for attribution}

Our main result is that timing is a very important source of information linking advertisements to conversions. We demonstrate this by fitting a novel point-process model to data on conversion and advertising across multiple channels, and showing that we can recover the true effect of advertising as calculated via randomized controlled trials (RCTs).

Multiple previous studies that used a ground truth (from simulations or from RCTs) to evaluate observational attribution methods have revealed significant inaccuracies. Methods evaluated in such a way include position-based approaches $[10,15]$ and data-driven approaches using either matching or propensity scoring $[4,14]$.

The method utilized in this report for attribution utilizes a probabilistic conversion model that heavily leverages timing information, similar to earlier published survival theory and Hawkes process models $[5,20,21]$. We were unable to rule out the accuracy of this model with RCTs. Since the main difference between this model and others that have been shown to be inaccurate is the heavy use of timing information, these results suggest that timing is highly informative for inferring attribution.

The importance of timing is also intuitively demonstrated in the correlograms of fig. 1 . We can easily see that not only is there a strong temporal correlation between advertisements and conversion events, but most of the correlation is concentrated just after impression time. This strong, punctuated correlation can be exploited by models (such as the approach presented here) that are designed to take advantage of it.

\subsection{The structure of the temporal interactions contains information}

The methods used in this paper are not the first to leverage timing data for inferring attribution, but they are the first to attempt to learn the structure of these correlations, rather than assuming a structure such as an exponential decay of influence.

Intuition for the value of learning the correlation structure is provided by the correlograms in fig. 2 . We can see that not only is the structure non-trivial, with both short and long timescale correlations differing between channels, but (as shown in fig. 2 B) even correlograms that appear simple do not follow a simple exponential decay.

The strongest evidence for the relevance of the correlation structure is demonstrated in fig. 5. This figure shows that a model with an assumed exponential decay in correlations (the "exponential model") fails to recover the true causal attribution as evaluated by RCT, while a model where the correlation structure is learned (the "full model") can.

\subsection{Phenomenological models allow for simulation and for inference}

The approach taken in this study (and several related previous studies, especially those based on temporal interactions $[5,16,21])$ can be seen as an example of phenomenological modeling. While the models used here are not derived from any first physical principles, their parameters are interpretable, and the models themselves can be used to simulate various scenarios.

One example of such use is to allocate credit for multi-touch attribution (MTA). We can do this by comparing realized conversions to the expected yield under various counterfactual scenarios. Results for such an analysis for the final treatment group (exposed to all ad types) in Experiment 1 are shown in fig. 6. For each channel, we calculate its contribution as the difference between the expected conversion yield with and without it. For comparison, we also show the incremental conversions given between RCT arms that add each channel.

While this treatment of MTA is not rigorous, and does not account for the ordering of channels, it illustrates how the approach can be applied. Some observations from this data are that the models allocate more evenly across the channels than the RCT data, and that the full model allocates less contribution to in-app ads than the exponential model (which is consistent with the exponential model's overestimate of the effect of in-app conversions in fig. 5). Future studies evaluating the suitability of this approach for MTA could include additional RCT arms that allow for channel importance measurement using the Shapley value [17], following Dalessandro et al. [3].

Another potential use of this type of phenomenological model is in optimizing the impression strategy. Since the model allows for a projection of the expected return on increased ad delivery, it could potentially also be used to find the amount of advertising that results in a desired yield to cost ratio. This would be useful for scenarios 
A
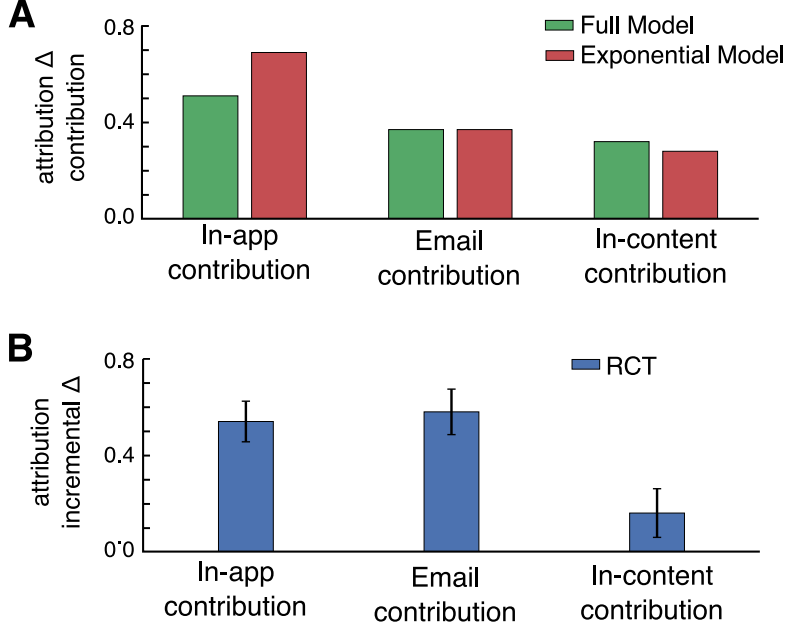

Figure 6: Estimate of effectiveness by advertising channel for Experiment 1. Panel $A$ shows a multi-touch attribution (MTA) channel breakdown for the full and exponential models, fit on data from the treatment group receiving promotions on all channels. Cf. Ji and Wang [5] fig. 3. While we cannot directly compare these MTA calculations to a "true" multi-touch attribution credit assignment, panel $B$ shows the incremental gain for each channel in the RCT for reference.

such as at Spotify, where ad delivery can be controlled directly with goals in mind, or for publishers who wish to give feedback to advertisers on what yield might be expected from increased spending.

\subsection{Caveats}

We have introduced a new temporal model for predicting conversions, and demonstrated that it can correctly infer the true causal attribution on experiments conducted with Spotify's advertising platform. These results, while novel, represent tests of a new method on a single platform. Since this platform's flexibility has simplified our experimental design and analysis in ways that may not be possible on other platforms, and since the modeling approach is not directly comparable to some others, we enumerate and discuss some differences here between our methodology and previous reports.

First, we were able to ensure that exposure to the promotional advertising was high for members of treatment groups (over $50 \%$ of users were exposed at least once). We could therefore avoid conditioning the conversion calculations on impressions. While this means we effectively underestimate the "true" conversion rate for both treatment and control groups (vs. what it would be if we only counted exposed treatment users), we avoid the difficult problem of finding appropriate control groups against only exposed users. Implementing this method on another platform where exposure rates were comparatively low would require using more sophisticated RCT analysis [6, 10].

Second, we chose as our metric the delta between the treatment and control group conversion rates. This simplified the analysis, so that confidence intervals could easily and reliably by determined. Some previous studies (e.g. [4]) use "lift" as a metric however. Lift, calculated as $\left(\mu_{G}-\mu_{G}^{C}\right) / \mu_{G}^{C}=\Delta_{G} /\left(\mu_{G}-\Delta_{G}\right)$, is a ratio whose numerator and denominator are both estimated from data. This means that small variability in $\Delta_{G}$ can correspond to a very large variability in lift (if conversion rates are low). Calculating confidence intervals for lift is also very difficult, since the numerator and denominator are not independent of each other. While the choice of the simpler metric should not affect the validity of the results, it does not highlight some of the metric uncertainty that exists for lift.

Finally, our modeling approach required that we use a small amount (5\%) of the control group for fitting models for the treatment groups in Experiments 1 and 2. This was necessary since the control group was exposed to generic ads which were not PSAs, and the treatment groups were not - we therefore had to include control data so that the model could learn parameters related to these generic ads. This was not necessary for Experiment 3, where control data was not used in fitting the model. We do not believe this use of control group data invalidates the results, since group assignment was not included as a feature (and hence the model was blind to it). The amount of control data used was also small: even if we labelled the data as control, the probability of finding a random $5 \%$ of the control group who could yield (by direct computation of the control conversion rate) an attribution measure within the 95\% CI for all the treatment groups in Experiments 1 and 2 is 0.0018 .

\subsection{Future Directions}

These results suggest that further research into observational attribution methods that make heavy use of temporal data could be fruitful. Since these results also suggest that models with an assumed fixed shape for temporal interactions (such as Hawkes processes) have some limitations, it is natural to suggest continued investigations into applying these types of flexible inhomogeneous Poisson models to the attribution problem.

One direction for such an investigation could be to continue to add reasonable interpretable parameters to linear-nonlinear feedforward models, as was done here (for instance, a future model could include factors to learn how different user populations respond to ads differentially). Another could be to incorporate recent methods that pair deep learning methods with inhomogeneous spiking output (such as the LFADS model [19]), which also attempt to uncover network dynamics.

\section{REFERENCES}

[1] D. Bond. Advertising agencies squeezed by tech giants. https://www.ft.com/ content/9a9ac60a-575a-11e7-9fed-c19e2700005f/, 2017. Accessed 2017-09-18.

[2] C. Brody. Correlations without synchrony. Neural Computation, 11(7):1537-1551, 1999.

[3] B. Dalessandro, O. Stitelman, C. Perlich, and F. Provost. Causally Motivated Attribution for Online Advertising. Proceedings of the Sixth International Workshop on Data Mining for Online Advertising and Internet Economy, 2012.

[4] B. R. Gordon, F. Zettelmeyer, N. Bhargava, and D. Chapsky. A Comparison of Approaches to Advertising Measurement: Evidence from Big Field Experiments at Facebook. SSRN Electronic fournal, 2017.

[5] W. Ji and X. Wang. Additional Multi-Touch Attribution for Online Advertising. Thirty-First AAAI Conference on Artificial Intelligence, 2017.

[6] G. A. Johnson, R. A. Lewis, and E. I. Nubbemeyer. Ghost Ads: Improving the Economics of Measuring Online Ad Effectiveness. fournal of Marketing Research, 2017. 
[7] L. Johnson. U.S. Digital Advertising Will Make $\$ 83$ Billion This Year, Says EMarketer. http://www.adweek.com/digital/ u-s-digital-advertising-will-make-83-billion-this-year-says-emarketer/, 2017. Accessed 2017-09-18.

[8] R. A. Lewis and J. M. Rao. The Unfavorable Economics of Measuring the Returns to Advertising. The Quarterly fournal of Economics, 130:1941-1973, 2015.

[9] R. A. Lewis, J. M. Rao, and D. H. Reiley. Here, There, and Everywhere: Correlated Online Behaviors Can Lead to Overestimates of the Effects of Advertising. Proceedings of the 20th ACM International World Wide Web Conference, 2011.

[10] R. A. Lewis, D. H. Reiley, T. A. Schreiner, C. Lewis, G. Ellison, J. Hausman, S. Ryan, and S. Ellison. Ad Attributes and Attribution: Large-Scale Field Experiments Measure Online Customer Acquisition. SSRN Electronic fournal, 2012.

[11] L. Paninski. Maximum likelihood estimation of cascade point-process neural encoding models. Comput. Neural Syst, 15:243-262, 2004.

[12] D. H. Perkel, G. L. Gerstein, and G. P. Moore. Neuronal Spike Trains and Stochastic Point Processes. Biophysical fournal, 7(4):391-418, 1967.

[13] J. W. Pillow, J. Shlens, L. Paninski, A. Sher, A. M. Litke, E. J. Chichilnisky, and E. P. Simoncelli. Spatio-temporal correlations and visual signalling in a complete neuronal population. Nature, 454(7207):995-999, 2008.
[14] S. Sapp and J. Vaver. Toward Improving Digital Attribution Model Accuracy. Technical report, Google, Inc, 2016.

[15] S. Sapp, J. Vaver, M. Shi, and N. Bathia. DASS: Digital Advertising System Simulation. Technical report, Google, Inc, 2016

[16] X. Shao and L. Li. Data-driven Multi-touch Attribution Models. Proceedings of the 17th ACM SIGKDD International Conference on Knowledge Discovery and Data Mining, pages 258-264, 2011.

[17] L. Shapley. A value for n-person games. Annals of Mathematics Studies, (28):307317, 1953.

[18] J. Shlens. Notes on Generalized Linear Models of Neurons. arXiv preprint arXiv:1404.1999, 2014

[19] D. Sussillo, R. Józefowicz, L. F. Abbott, and C. Pandarinath. LFADS - latent factor analysis via dynamical systems. CoRR, abs/1608.06315, 2016.

[20] L. Xu, J. A. Duan, and A. Whinston. Path to Purchase: A Mutually Exciting Point Process Model for Online Advertising and Conversion. Management Science, 60(6):1392-1412, 2014.

[21] Y. Zhang, Y. Wei, and J. Ren. Multi-Touch Attribution in Online Advertising with Survival Theory. IEEE International Conference on Data Mining, 2014. 